\title{
Hybrid particle-field molecular dynamics simulations of charged amphiphiles in aqueous environment
}

\author{
Hima Bindu Kolli, ${ }^{\dagger}$ Antonio de Nicola, ${ }^{\ddagger}$ Sigbjørn Løland Bore ${ }^{\dagger}$ Ken Schäfer, ${ }^{\S}$ \\ Gregor Diezemann, ${ }^{\S}$ Jürgen Gauss, ${ }^{\S}$ Toshihiro Kawakatsu, $\|$ Zhong-Yuan Lu, ${ }^{\perp}$ \\ You-Liang Zhu,, Giuseppe Milano,,$\stackrel{*}{ }$ Michele Cascella" ${ }^{*}$
}

$\uparrow$ Department of Chemistry and Hylleraas Centre for Quantum Molecular Sciences, University of Oslo, P.O. Box 1033 Blindern, 0315 Oslo, Norway

† Department of Organic Materials Science, Yamagata University, 4-3-16 Jonan Yonezawa, Yamagata-ken 9928510, Japan

$\S$ Institut für Physikalische Chemie, Johannes Gutenberg-Universität Mainz, Duesbergweg 10-14, 55128 Mainz, Germany

|| Department of Physics, Tohoku University, Aoba, Aramaki, Aoba-ku, Sendai 980-8578, Japan

$\perp$ State Key Laboratory of Supramolecular Structure and Materials, Institute of Theoretical Chemistry, Jilin University, Changchun 130023, China

\# State Key Laboratory of Polymer Physics and Chemistry, Changchun Institute of Applied Chemistry, Chinese Academy of Sciences, Changchun 130022, China.

*email to: gmilano@yz.yamagata-u.ac.jp,michele.cascella@kjemi.uio.no 


\begin{abstract}
We develop and test specific coarse-grained models for charged amphiphilic systems such as palmitoyloleoyl phosphatidylglycerol (POPG) lipid bilayer, and sodium dodecyl sulphate (SDS) surfactant in aqueous environment, to verify the ability of the hybrid particle-field method to provide a realistic description of polyelectrolyte soft matter systems. According to the hybrid approach, the intramolecular interactions are treated by a standard molecular Hamiltonian and the non-electrostatic intermolecular forces are described by density fields. Electrostatics is introduced as an additional external field obtained by a modified particle-mesh Ewald procedure, as recently proposed in [Phys. Chem. Chem. Phys 2016, 18, 9799]. Our results show that, upon proper calibration of key parameters, electrostatic forces can be correctly reproduced. Molecular dynamics simulations indicate that the methodology is robust with respect to the choice of the relative dielectric constant, yielding the same correct qualitative behavior for a broad range of values. In particular, our methodology reproduces well the organization of the POPG bilayer, as well as the SDS concentration-dependent change in the morphology of the micelles from spherical to microtubular aggregates. The inclusion of explicit electrostatics with good accuracy and low computational costs paves the way for a significant extension of the hybrid particle field method to biological systems, where the polyelectrolyte component plays a fundamental role for both structural and dynamical molecular properties.
\end{abstract}




\section{Introduction}

Amphiphiles are macromolecules characterized by well-defined hydrophobic and hydrophilic regions. Depending on the molecular shape and on the hydrophobic/hydrophilic balance, they tend to selfassemble into a variety of aggregates, like micelles and vesicles, lamellar structures like mono- and bilayers and structures with high aspect ratio like nanotubes and nanofibers. ${ }^{1-4}$ These supramolecular structures are used in many industrial and biotechnological processes such as drug delivery systems, ${ }^{5}$ micro- and nanoscale micellar reactors, ${ }^{6}$ dispersants, detergents, emulsifiers, and coatings. The determination of the supramolecular structural organization of the amphiphilic molecule depends on a variety of factors, including the shape and flexibility of the single molecular structure the interplay of intra- and inter-molecular forces, and external thermodynamic factors like temperature, pressure, concentration, or ionic strength. Due to such complexity, the prediction and determination of the shape and size of amphiphiles, as well as their aggregation mechanisms, remains a challenging task both for experimentalist and computational scientists.

In recent times, scattering techniques such as small-angle X-ray scattering (SAXS) ${ }^{7}$ or small-angle neutron scattering (SANS) ${ }^{8,9}$ have been widely used for the quantitative characterization of the shape, internal structure, and interactions of macromolecular aggregates, like protein complexes in solution $^{10,11}$ as well as for micellar transformation studies. ${ }^{12}$ Light scattering techniques are widely used to determine the shape of micelles in solution and Fluorescent spectroscopy is used to measure the aggregation number and the critical micelle concentration (CMC). Unfortunately, these techniques, having shortest temporal resolutions in the order of the millisecond, are not fast enough to capture the structural changes during the aggregation process. ${ }^{13}$ Moreover, at lower concentrations, the interplay between shape variations and polydispersity complicates the data interpretation and could lead to ambiguous conclusions regarding the shape of surfactant micelles.

The picture is even more complex when studying biological amphiphilic systems like bio-membranes. Such bilayer structures present a chemically complex composition, being mostly formed by mixtures of phospholipids, with the addition, at very diverse stoichiometric ratios, of a variety of sphingolipids, cholesterol and the presence of both peripheral and transmembrane proteins. ${ }^{14,15}$ Due to both their chemical complexity and their disordered nature, it is hardly possible to follow their dynamics and interactions in detail at the atomistic level. ${ }^{16,17}$

Computer modeling offers, in principle, an effective complementary way of exploring self-assembly processes at molecular resolution, and at highly controlled thermodynamic and stoichiometric conditions. Unfortunately, the assembling process occurs at the mesoscopic time- $(>\mu$ s) and length- 
scales $(>100 \mathrm{~nm})$, which are computationally expensive in a molecular dynamics framework employing explicit all-atom models. ${ }^{18-24}$ For this reason, the use of coarse-grained (CG) simulations has become an alternative that aims to bridge the time and length scales involved in self-assembly phenomena.

The underlying assumption for the study of self-assembly phenomena at the CG scale is that neither the molecular structure at atomistic length scales nor its fast motion are relevant to the dynamics of the slow aggregation process. Therefore, molecular moieties can be conveniently described by a smaller number of order parameters considering just key properties like the amphiphilic nature of the individual molecular segments. CG models in both explicit and implicit solvent display a great potential for capturing critical phase behaviour of surfactants, phospholipid bilayers and generally of soft matter, as demonstrated, for example, in several studies available in the literature. ${ }^{25-35}$

However, standard CG approaches are subject to analogous technical computational bottlenecks of all atom models, produced by the need of the evaluation of distance-dependent intermolecular interactions. ${ }^{36}$ As a consequence, unless massively parallel architectures are available, common CG simulations are limited to systems with sizes not larger than $100 \mathrm{~nm}$ length- and $\sim 1-100 \mu$ s time scales. ${ }^{37,38}$ These numbers are still too small compared to mesoscopic dimensionalities with length scales in the order of 100-1000 $\mathrm{nm}$ and time scales in the order of the millisecond. ${ }^{39}$

An alternative approach which is not bound to such limitation is the continuous field representation. In the framework of self-consistent field theory, the model systems are not represented by particles but by density fields and the mutual interactions between segments are decoupled and replaced by interactions with static external fields. These field-based approaches allow to simulate materials on scales much larger than the ones attainable with particle-based simulations. In recent years, the hybrid particle-field (hPF) approach combining a microscopic molecular representation to density-based potential has been introduced. $^{40-42}$ In particular, the determination of an analytical expression for local potential energy gradients, and consequently for the forces acting on the individual particles, allowed Milano and Kawakatsu to reformulate the hPF method within a molecular dynamics (MD) framework (hPF-MD hereafter). ${ }^{43,44}$ The hPF-MD method was validated for different molecular models including molecular surfactants, atomistic models of polymers and bio membranes. ${ }^{45-51}$

More recently, an efficient electrostatic treatment based on the Ewald summation in the framework of hPF-MD has been proposed by Zhu et al. ${ }^{52}$ Like for the density field, charged molecules are interacting with an external electrostatic field derived from the charge density. The long-range part of the Coulomb interaction is evaluated in reciprocal space using Fourier series just like in the standard particle-mesh Ewald method. The short-range part is evaluated by collecting the contributions of short range energy from the surrounding charges and matching with the Flory Huggins interaction parameter.

The explicit treatment of electrostatics opens up the possibility of using hPF schemes to investigate 
major biological processes that are dominated by such interactions - for example ion-membrane permeation, membrane electroporation, protein/protein and protein/membrane interactions, or assembly and dynamics of nucleic acids. In fact, the hPF-MD method with electrostatics has been validated against particle-based simulations for model polyelectrolytes only. ${ }^{52}$ In this work, we present the first application of hPF-MD to realistic soft matter models. The aim of the paper is to validate the models for charged amphiphile systems. Specifically, we investigated the ability of hPF-MD in describing both the structural properties of a constituted charged palmitoyloleoyl phosphatidylglycerol (POPG) bilayer, and the aggregation dynamics of sodium dodecyl-sulphate (SDS) in water. Our analysis provides a critical assessment of the quality performances of hPF-MD as a function of different external parameters, like the dielectric constant and $\chi$ parameters defining the effective interactions between particles and density field. The hPF-MD has been implemented in both serial and parallel versions of the OCCAM code. ${ }^{47}$

In section 2.1, a brief description of hPF-MD method is given. The treatment of the short- and longrange parts of the electrostatics interaction in hPF-MD is shown in the section 2.2. In section 2.3, the POPG and SDS models along with simulation details are given respectively. We discuss the calibration of the Ewald convergence parameter in section 3.1 and the simulation results on POPG bilayer and SDS aggregation are presented and discussed in sections 3.2 and 3.3. Final remarks and conclusions are presented in section 4 .

\section{Methods}

\subsection{The Hybrid Particle-Field approach}

The hPF-MD method and its application to coarse-grained and atomistic models has been introduced in a series of former publications. ${ }^{43-48,50,51}$ Here we only briefly recall the main ideas. In hPF-MD, a discrete particle-based representation of several molecules interacting through pair forces between nonbonded particles, is formally transformed into a set of decoupled molecules subject to an external potential depending on density fields.

Under this transformation and considering the following functional form for the potential energy,

$$
W[\{\phi(\boldsymbol{r})\}]=\frac{1}{\phi_{0}} \int d \boldsymbol{r}\left(\frac{k_{B} T}{2} \sum_{i j} \chi_{i j} \phi_{i}(\boldsymbol{r}) \phi_{j}(\boldsymbol{r})+\frac{1}{2 \kappa}\left(\sum_{i} \phi_{i}(\boldsymbol{r})-\phi_{0}\right)^{2}\right)
$$


applying the saddle point approximation, by functional differentiation it is possible to obtain the meanfield external potential $V_{i}^{\text {ext }}(\boldsymbol{r})$ acting on an individual particle of type $i$ at position $\boldsymbol{r}$, which takes the form:

$$
V_{i}^{e x t}(\boldsymbol{r})=\frac{\delta W[\{\phi(\boldsymbol{r})\}]}{\delta \phi_{i}(\boldsymbol{r})}=\frac{1}{\phi_{0}}\left(k_{B} T \sum_{j} \chi_{i j} \phi_{j}(\boldsymbol{r})+\frac{1}{\kappa}\left(\sum_{j} \phi_{j}(\boldsymbol{r})-\phi_{0}\right)\right)
$$

In equations $1,2 k_{B}$ is the Boltzmann constant, $T$ is the temperature of the system, $\phi_{i}(\boldsymbol{r})$ is the coarsegrained number density of the species $i$ at position $r, \chi_{i j}$ is the mean field interaction strength between particles of type $i$ and $j, \kappa$ is a compressibility parameter acting against local density inhomogeneity, and $\phi_{0}$ is the total number density of the system.

The forces acting on the individual particles are computed as the spatial gradients of the same potential in equation 2 . The density field and its gradient are computed on a spatial grid and updated with a time interval $\tau>\Delta t$, where $\Delta t$ is the MD time-step. This ensures fast computation of forces, in an embarrassingly parallel implementation. More details about the derivation of equation 2 and its implementation in MD simulations are reported in references ${ }^{43,44,47}$.

\subsection{The hybrid Particle-Field method with electrostatics}

The electrostatic potential $\psi(\boldsymbol{r})$ produced by a distribution of charges $\rho(\boldsymbol{r})$ is computed by solving the Poisson equation:

$$
-\nabla^{2} \psi(\boldsymbol{r})=\frac{\rho(\boldsymbol{r})}{\varepsilon_{r} \varepsilon_{0}}
$$

where $\varepsilon_{0}$ is the vacuum permittivity, and $\varepsilon_{r}$ is the relative dielectric constant of the medium. Adopting periodic boundary conditions, the solution of the equation 3 yields the potential field $\psi(\boldsymbol{r})$ :

$$
\psi(\boldsymbol{r})=\frac{1}{4 \pi \varepsilon_{r} \varepsilon_{0}} \sum_{n} \sum_{j=1}^{N} \frac{q_{j}}{\left|\boldsymbol{r}-\boldsymbol{r}_{\boldsymbol{j}}+\boldsymbol{n} \boldsymbol{L}\right|}
$$

Here, the vector $\mathbf{L}=\left(\mathbf{L}_{1}, \mathbf{L}_{2}, \mathbf{L}_{3}\right)$ defines the simulation box, and $n \mathbf{L}=\mathrm{n}_{1} \mathbf{L}_{1}+\mathrm{n}_{2} \mathbf{L}_{2}+\mathrm{n}_{3} \mathbf{L}_{3}$ is the translation over a generic periodic image. Recently, an efficient method based on the particle-mesh Ewald summation was implemented in hybrid particle field simulations to compute the electrostatic potential shown in equation $4 .^{47}$ The short-range and long-range parts of the electrostatic potential in conventional Ewald sum are written as:

$$
\begin{aligned}
& \psi^{S}(\boldsymbol{r})=\frac{1}{4 \pi \varepsilon_{0} \varepsilon_{r}} \sum_{n} \sum_{j} \frac{q_{j} \operatorname{erfc}\left(\alpha\left|\boldsymbol{r}-\boldsymbol{r}_{\boldsymbol{j}}+n \boldsymbol{L}\right|\right)}{\left|\boldsymbol{r}-\boldsymbol{r}_{\boldsymbol{j}}+n \boldsymbol{L}\right|} \\
& \psi^{L}(\boldsymbol{r})=\sum_{m \neq 0} \hat{\psi}^{L}(\mathbf{m}) \exp (i \boldsymbol{m} \cdot \boldsymbol{r})
\end{aligned}
$$

where $\psi^{S}$ is the short-range part, and $\psi^{L}$ is the long-range part of the electrostatic potential. $\hat{\psi}^{L}$ 
denotes $\psi^{L}$ in the reciprocal space, and it is given by:

$$
\widehat{\psi}^{L}(\boldsymbol{m})=\frac{\exp \left(-\mathbf{m}^{2} / 4 \alpha^{2}\right)}{\varepsilon_{0} \varepsilon_{r} V \mathbf{m}^{2}} \sum_{j=1}^{N} q_{j} \exp \left(-i \boldsymbol{m} \cdot r_{j}\right)
$$

where $\mathrm{V}$ is the volume of the simulation box, $\mathbf{m}$ is the reciprocal space vector, and $\alpha$ is the Ewald's convergence parameter.

In hPF-MD, the charge density is expressed in terms of $Q(\boldsymbol{l})=Q\left(l_{1}, l_{2}, l_{3}\right)$, the distribution of charges at the lattice points. The long-range part of the electrostatic potential at the lattice point $\boldsymbol{l}$, is computed using forward $(F)$ and backward $\left(F^{-1}\right)$ discrete Fourier transforms:

$$
\begin{aligned}
\psi^{L}(\boldsymbol{l}) & =\sum_{m_{1}=0}^{N_{1}-1} \sum_{m_{2}=0}^{N_{2}-1} \sum_{m_{3}=0}^{N_{3}-1} \hat{\psi}^{L}(\boldsymbol{m}) \exp \left[2 \pi i\left(\frac{m_{1} l_{1}}{N_{1}}+\frac{m_{2} l_{2}}{N_{2}}+\frac{m_{3} l_{3}}{N_{3}}\right)\right] \\
& =F^{-1}\left[\left(\frac{\exp \left(\frac{-\boldsymbol{m}^{2}}{4 \alpha^{2}}\right)}{\varepsilon_{0} \varepsilon_{r} V \boldsymbol{m}^{2}}\right) F Q\right]\left(l_{1}, l_{2}, l_{3}\right)
\end{aligned}
$$

The short-range part of the electrostatic potential at the lattice point of spatial position $\boldsymbol{l}$ is given by:

$$
\psi^{S}(\boldsymbol{l})=\frac{z_{C N}}{4 \pi \varepsilon_{0} \varepsilon_{r}} \frac{\operatorname{erfc}(\alpha \sigma)}{\sigma} Q(\boldsymbol{l})
$$

where the parameter $\mathrm{z}_{\mathrm{CN}}$ is the coordination number which takes a value of 6 for a three-dimensional cubic lattice and $\sigma$ is the particle diameter. Instead of the pairwise short-range electrostatic interactions like in classic Ewald summation, the interactions are now transmitted through the mean field.

The performance of the hPF-MD method has been studied thoroughly and documented in ref. 47. Since the density fields vary slowly with respect to the particle motions, they are, with negligible errors, updated in the intervals of multiple time steps. Several benchmark studies were performed changing the density update frequency and the grid size. Compared to MARTINI simulations, the hPF-MD method performs $\sim 15$ times faster with an update frequency of $100 \mathrm{MD}$ steps and 32,768 grid points. In the new implementation, the additional computational costs are due to the fast Fourier transform (FFT) routine, and add roughly $7 \%$ to the total simulation time.

\subsection{Simulation Details}

2.3.1 POPG bilayer The POPG bilayer model was composed of 1216 POPG lipids (608 lipids for each 
layer), 64448 water beads, each with a density of 4 water molecules, and $1216 \mathrm{Na}^{+}$counterions necessary for charge neutralization in a $20.18 \times 20.18 \times 23.29 \mathrm{~nm}^{3}$ periodic box. The CG mapping of POPG is explained in Figure 1. Each POPG has a polar head (P) with net charge -1 . The bead type D mimics the presence of a double-bond in one of the aliphatic tails. The intramolecular parameters for POPG were taken from the literature. ${ }^{25,53}$

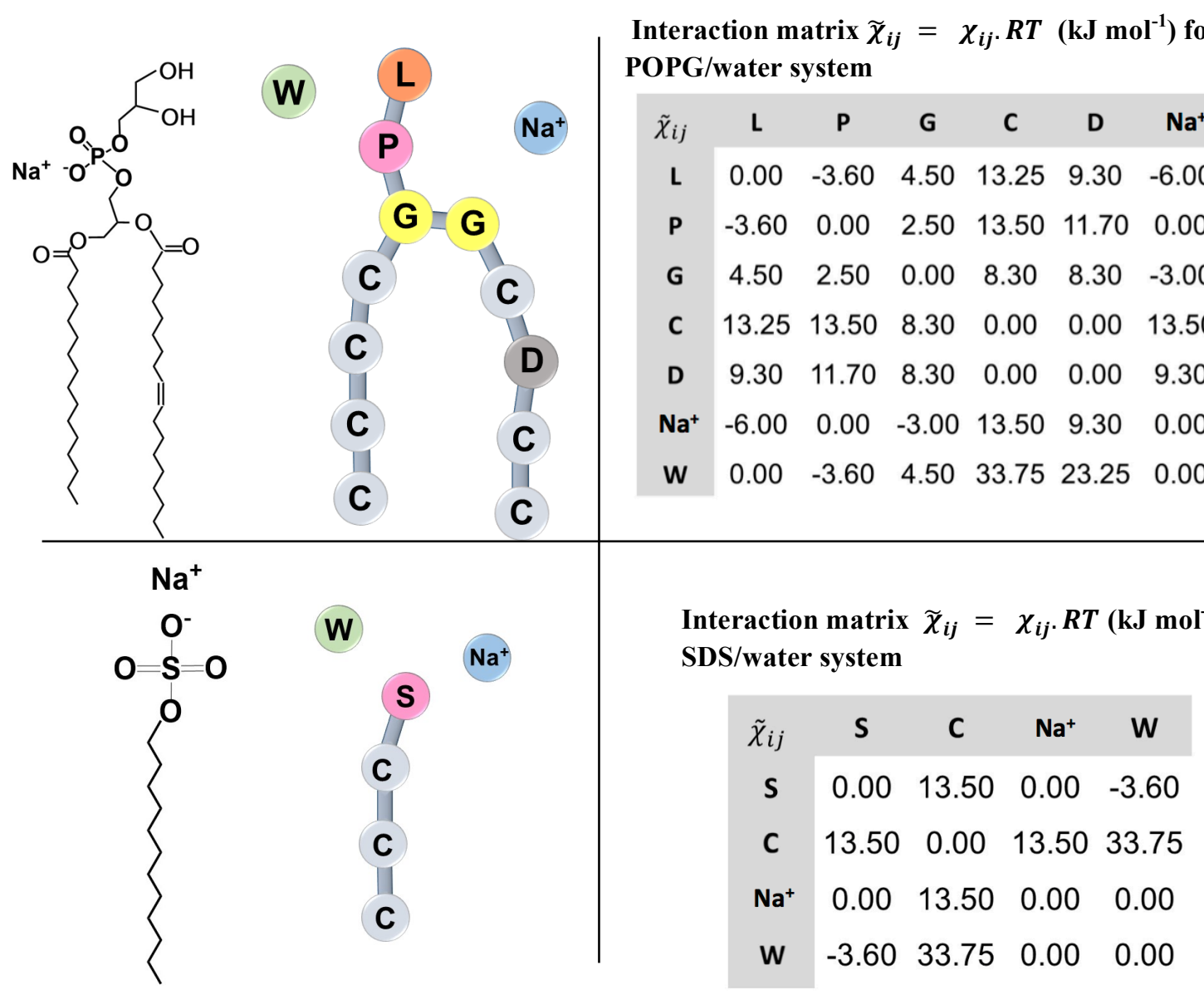

Figure 1. CG model and particle field interaction parameters of POPG/water and SDS/water. Top left: Structure of POPG and CG mapping. Top right: Table showing the particle-field interaction parameters for the POPG/ water system. Bottom left: Structure of SDS and CG mapping. Bottom right: Table showing the particle-field interaction parameters for the SDS/water system.

First, the POPG bilayer was relaxed over $2 \mu$ s of particle-particle (PP) CG simulations, using the MARTINI force field. ${ }^{53}$ The CG simulations were run in the NPT ensemble at $301.15 \mathrm{~K}$ and $1 \mathrm{bar}$, using semi-isotropic pressure coupling. Electrostatic interactions were computed via particle-mesh Ewald summation, using a dielectric constant $\varepsilon_{\mathrm{r}}=15$, and a grid of $0.3 \mathrm{~nm}$ mesh. A cut-off of $1.5 \mathrm{~nm}$ was used to truncate the van der Waals terms. The PP-CG simulations were run using the GROMACS 4.5.4 package. $^{54}$ 
The final configuration, obtained from the PP-CG run, was used as the starting configuration for the hPF-MD simulations. All intramolecular interactions were treated in the same manner as in the PP-CG simulations. The particle field parameters $\tilde{\chi}_{i j}=\chi_{i j} R T$ (where $\mathrm{R}$ is the gas constant) needed to calculate the intermolecular interactions between particle types $i$ and $j$ are listed in figure 1 . The value of the incompressibility parameter $\kappa^{-1}$ was set to $4 \mathrm{RT}$, in agreement with previous simulations of water/lipid mixtures. ${ }^{45,46,48}$ We consider the same grid size $(32 \times 32 \times 36)$ for both particle and charge fields. The size of the grid has been chosen to keep the cell length, $b \approx 0.65 \mathrm{~nm}$ and the explanation for this choice is given in section 3.1. The hPF-MD simulations were run using a time step of $0.03 \mathrm{ps}$ in NVT ensemble, at a temperature of $301.15 \mathrm{~K}$ using an Andersen thermostat ${ }^{55}$ with collision frequency of $7 \mathrm{ps}^{-1}$. A density-field update time interval $\tau=100 \Delta t$ was employed for both particle and charge densities.

2.3.2 SDS aggregation The SDS molecules were represented by a chain of four beads as shown in Figure 1. Each chain consists of one hydrophilic polar head bead (S) with a charge of -1 charge units and three hydrophobic beads (C1, C2 and C3), each one representing four $\mathrm{CH} 2 / \mathrm{CH} 3$ units. The SDS bonded parameters are taken from ref. ${ }^{56}$ The interaction matrix $\tilde{\chi}_{i j}$ used for hPF-MD is given in Figure 1. The value of the compressibility was set to $\kappa^{-1}=4 \mathrm{RT}$. The density update was set to $\tau=100 \Delta t$. The hPF-MD simulations were performed at four different SDS concentrations: $\mathrm{C}_{\mathrm{SDS}}=50 \mathrm{mM}, 72$ $\mathrm{mM}, 182 \mathrm{mM}$ and $400 \mathrm{mM}$, using a cubic periodic box of edge $33.2 \mathrm{~nm}$, containing 1120, 1600, 4000, and 8815 SDS molecules, respectively. The chosen concentrations are all significantly higher than the $\mathrm{CMC}$ for SDS, which is $8 \mathrm{mM}$. An equal number of $\mathrm{Na}^{+}$beads were added to act as counterions; roughly $\sim 300,000$ water beads were used in all simulations. All the four systems and their compositions are shown in table 1 . We consider a grid size of $50 \times 50 \times 50$ for both charge and particle fields.

Initially, SDS, $\mathrm{Na}^{+}$, and water were placed at random positions in the simulation box. This initial condition resembles the ideal limit of the experimental condition in which fast mixing techniques are used in preparing the system. All MD runs were performed in the NVT ensemble using $\Delta t=0.03 \mathrm{ps}$ keeping the temperature constant at $298 \mathrm{~K}$ using the Andersen thermostat ${ }^{55}$ with a collision frequency of $7 \mathrm{ps}^{-1}$.

All the hPF-MD simulations of both POPG and SDS systems were run using the parallel version of OCCAM code. $^{47}$ 
Table 1. SDS systems simulated and the composition of the systems

\begin{tabular}{ccccc}
\hline C $\mathrm{SDS}$ & $\begin{array}{c}\text { Total no. of } \\
\text { particles in the box }\end{array}$ & $\begin{array}{c}\text { Number of } \\
\text { SDS molecules }\end{array}$ & $\begin{array}{c}\text { No. of } \\
\mathrm{Na}^{+} \text {ions }\end{array}$ & $\begin{array}{c}\text { No. of } \\
\text { water beads }\end{array}$ \\
\hline $50 \mathrm{mM}$ & 309600 & 1120 & 1120 & 304000 \\
$72 \mathrm{mM}$ & 304000 & 1600 & 1600 & 296000 \\
$182 \mathrm{mM}$ & 306000 & 4000 & 4000 & 286000 \\
$400 \mathrm{mM}$ & 317075 & 8815 & 8815 & 273000
\end{tabular}

\section{Results and discussion}

\subsection{Calibration of Ewald parameters and grid size}

The hPF-MD implementation relies on a modified particle-mesh Ewald treatment to determine the short- and long-range components of the electrostatic potential. The accuracy of this approach is dependent on the grid spacing and the spread of the auxiliary Gaussian charge distribution $\alpha$ (equation 7). To assess this dependence, we used a model system comprised of two oppositely unit charged particles at a distance $\mathrm{d}$ along the $\mathrm{z}$-axis in a large simulation box of edge $\mathrm{L}=20.0 \mathrm{~nm}$. Figure 2 reports the intensity of the electrostatic forces acting on the two charges as a function of the grid spacing and $\alpha$, as well as the theoretical Coulomb force $\mathrm{F}_{\mathrm{C}}$. The profiles indicate that for values of $\alpha$ larger than $2.35 \mathrm{~nm}^{-1}$, our implementation consistently reports similar values of the forces. For a grid of $0.65 \mathrm{~nm}$,

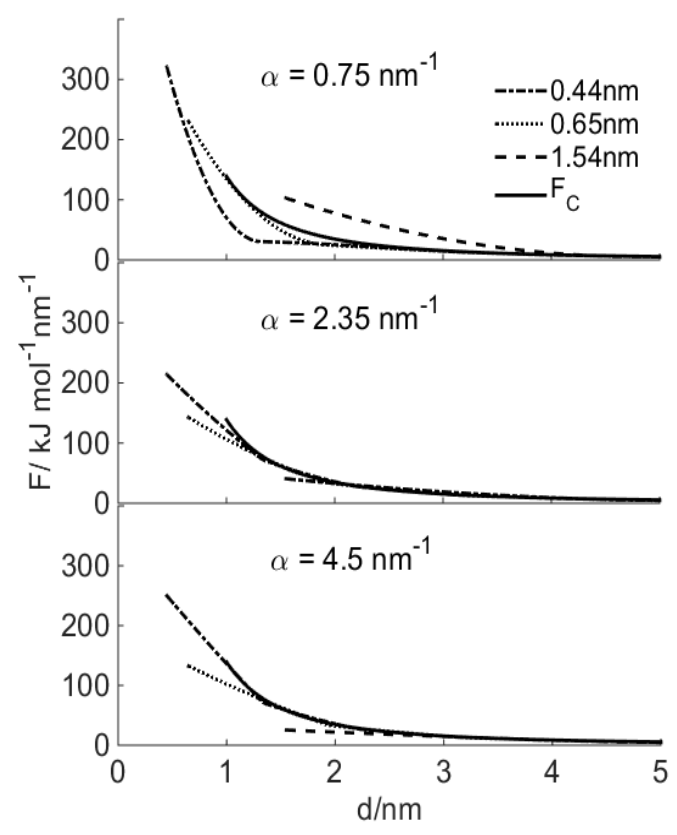

Figure 2. Electrostatic forces acting on two unit charges as a function of the distance between charges using different $\alpha$ values, and different grid spacing as reported in the legend. Top: electrostatic force for $\alpha=$ $0.75 \mathrm{~nm}^{-1}$. Middle: electrostatic force for $\alpha=2.35 \mathrm{~nm}^{-1}$. Bottom: electrostatic force for $\alpha=4.5 \mathrm{~nm}^{-1}$. All values are compared to the theoretical Coulomb force $\mathrm{F}_{\mathrm{C}}$. 
the corresponding average relative errors are 4\%, while for smaller $\alpha$, the error on the forces increases to $10 \%$. This is due to the fact that the Gaussian spread of the charge is inconsistently represented by the actual distribution of the charges on the mesh points. Furthermore, by grid refinement to a spacing of $0.45 \mathrm{~nm}$, greater accuracy, especially at short distances, is achieved with an average relative error of $3 \%$. In agreement with these findings, all the simulations were run using a value of grid spacing of 0.65 $\mathrm{nm}$ and $\alpha=2.35 \mathrm{~nm}^{-1}$.

\subsection{POPG bilayer}

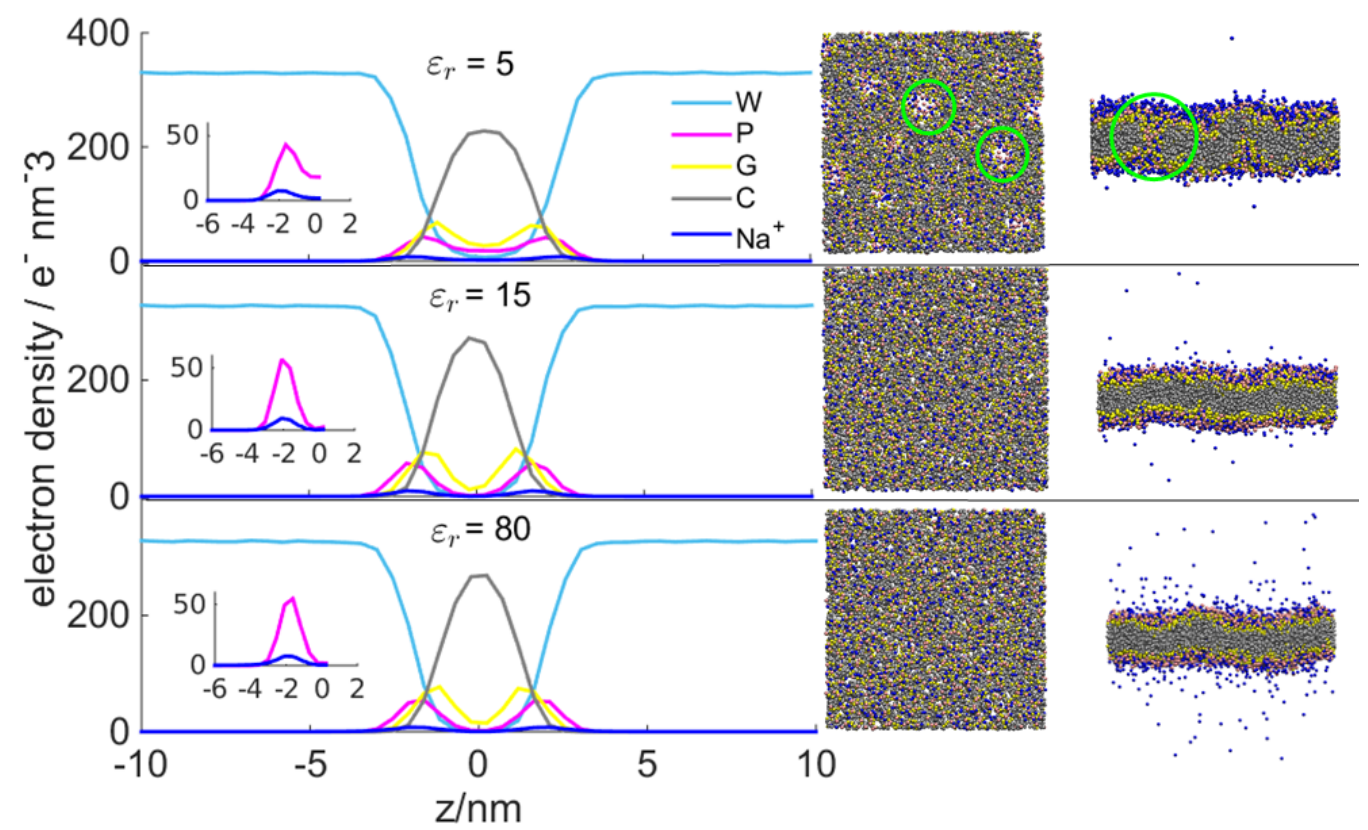

Figure 3. Left: The number density profile of POPG, $\mathrm{Na}^{+}$and water obtained from $1 \mu \mathrm{s}$ long hPF-MD simulations with $\varepsilon_{\mathrm{r}}=5,15$, and 80. Right: Snapshots with top and side-view of the bilayers. Water is not shown for clarity. The green circles evidence the formation of pores.

The bilayer structure of POPG was simulated using different values of the relative dielectric constant $\varepsilon_{\mathrm{r}}$ in the NVT ensemble by hPF-MD simulations. Figure 3 shows the number density profile of the POPG/water system at $\varepsilon_{r}=5,15$ and 80 obtained from the last $1 \mu$ s out of a $2 \mu$ s long hPF-MD run along the direction normal to the plane of the bilayer. For very small values of $\varepsilon_{r}$, we observed the formation of strong ion pairs between the positively charged $\mathrm{Na}^{+}$ions and the negatively charged $\mathrm{P}$ beads of POPG. The localization of $\mathrm{Na}^{+}$in the neighborhood of the lipid heads reduced drastically the effective charge at the surface of the lipids, thus enhancing the tendency of POPG to aggregate. As a result, the lateral tension of the membrane produced poration of the membrane.

In fact, the organization of POPG in a self-assembled structure is the result of the balance among competing interactions, in particular, the attractive collapse of the hydrophobic tails, the electrostatic 
repulsion of the charged heads, and the entropy. The electrostatic repulsion is screened by the formation of ion pairs, thus favoring the collapse of the lipids. At NVT conditions, and in periodic boundary conditions, stable flat bilayers are observed only if the global free-energy balance between such interactions produces POPG aggregates characterized by a surface area-per-lipids close to a value $A=N_{P O P G} /\left(L_{i} L_{j}\right)$, where $N_{P O P G}$ is the number of lipids in one leaflet, and $L_{i}, L_{j}$ are the lengths of two edges of the box.

Values of $\varepsilon_{r}$ higher than 10 yielded a stable bilayer structure. In these cases, the lipid moiety showed an organized lamellar structure, with a symmetric distribution of the components in the two leaflets. Overall, the thickness of the bilayer $\mathrm{D}_{\mathrm{HH}}$ appears less pronounced than in other molecular models, ${ }^{57-60}$ and in very good agreement with experimental data (Table S1, in Supporting Information (SI)). The rather broad distribution of the $\mathrm{G}$ beads indicates that the membrane is quite disordered, with appearing fast-dissipating undulations, clearly observable by visual inspection of the simulation trajectory. The appearance of local distortions is correlated to the binding dynamics of the $\mathrm{Na}^{+}$counterions on the membrane surface. As expected, the increase in the dielectric constant, and the consequent weakening of the electrostatic forces, results into a reduced adsorption of the counterions (Figure S1, SI), and a different binding dynamic for the ions.

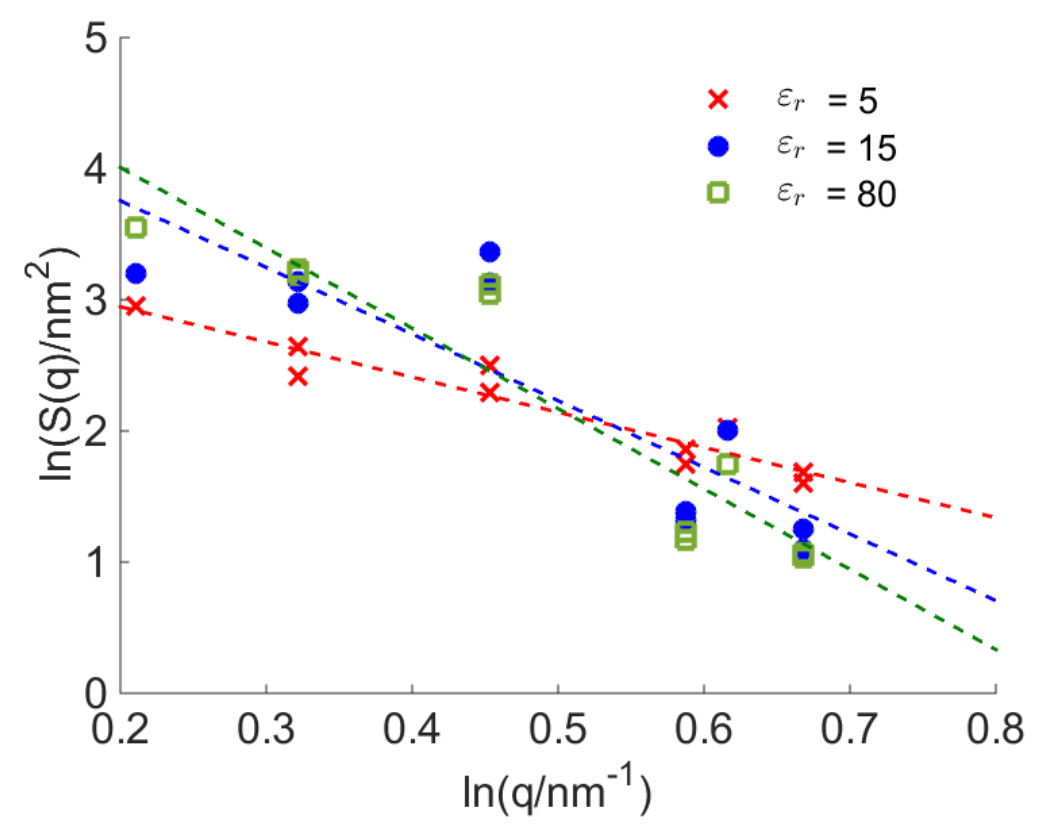

Figure 4. Frequency spectrum of POPG undulations. Intensity of the fluctuation frequencies as a function of the wave number q obtained from hPF-MD simulations using three different values of $\varepsilon_{\mathrm{r}}=5,15,80$.

At very low value of the dielectric constant, we observe different shape fluctuations of the membrane. A quantitative measure of such distortions can be obtained using the Helfrich continuum model ${ }^{61-65}$, as explained in detail in SI. 
Figure 4 reports the fluctuation spectrum of the POPG membrane computed over the last $100 \mathrm{~ns}$ of the simulations. In all systems, undulations dominate at wavenumbers $q$ smaller than $1.5 \mathrm{~nm}^{-1}$. Nonetheless, simulations at $\varepsilon_{r}=5$ show a suppression of the low-frequency modes, and some activation of modes at higher frequency compared to simulations at $\varepsilon_{r}=15$, and 80 . Simulations at $\varepsilon_{r}=15$ and 80 show instead a different distribution of the amplitude in the low-frequency regions, in particular, with the activation of fluctuations at wavenumbers $\left(\mathrm{q}<2 \mathrm{~nm}^{-1}\right)$, with similar values of the amplitudes. This confirms that the morphology of the membrane is stable in a broad range of dielectric values, with the increased screening of the dielectric compensating for the unbinding of the ions from the membrane surface.

\subsection{SDS Self-assembly}

We simulated four different SDS/water systems with SDS concentrations $\mathrm{C}_{\mathrm{SDS}}=50 \mathrm{mM}, 72 \mathrm{mM}, 182$ $\mathrm{mM}$, and $400 \mathrm{mM}$ for different values of $\varepsilon_{r}$, over up to $5 \mu \mathrm{s}$, starting from a randomly dispersed distribution of the SDS molecules and the $\mathrm{Na}^{+}$ions in water. Figure 5 shows the average aggregation number of SDS $\left\langle N_{a g g}\right\rangle$ plotted with respect to time. $N_{a g g}$ is computed by counting the number of micelles and the molecules in each micelle with cut-off distance $1.7 \sigma$ for each configuration using the linked list algorithm. The cut-off distance was defined as $0.8 \mathrm{~nm}$, corresponding to the first minimum of the radial distribution function between the P-N species. In all cases, we observed an almost instantaneous formation of small SDS aggregates, corresponding to a continuous increase of $N_{\text {agg. }}$ With time, such small moieties fuse together to form progressively larger and larger micelles. These events correspond to a continuous rather than step-wise increase of the $N_{\text {agg }}$ value.

The size and the shape of the micelle are directly dependent on the SDS concentration and the value of the dielectric constant. In particular, for medium values of the dielectric constant $\left(\varepsilon_{r}=45\right)$, regular spherical micelles were formed when the concentration of SDS was relatively low $\left(\mathrm{C}_{\mathrm{SDS}}=50,72 \mathrm{mM}\right)$. Increasing the SDS concentration $\left(\mathrm{C}_{\mathrm{SDS}}=182 \mathrm{mM}\right)$ led to spheroidal micelles with a prolate character, and eventually to the formation of nanotubular structures $\left(\mathrm{C}_{\mathrm{SDS}}=400 \mathrm{mM}\right)$, in agreement with experiment, and previous calculations using different CG models. ${ }^{66-70}$ In particular, the morphological transformation from spherical to microtubular micelles observed in our simulations reproduces the one previously observed using the MARTINI CG model, both with explicit or implicit solvation, ${ }^{68-69}$ as well as dissipative particle dynamics simulations. ${ }^{70}$ SDS aggregation is sensitive to the choice of the dielectric constant. In fact, higher values of the dielectric constant $\left(\varepsilon_{r}=80\right)$ yielded smaller micelles 
that are of spherical shape also at higher concentrations of SDS. For smaller values of $\varepsilon_{r}(\sim 15)$, the hPFMD simulations did not produce regular assemblies, but systematically predicted a phase of irregularlydispersed aggregated surfactants, which does not correspond to any experimentally observed assembly. Direct influence of the choice of the dielectric on the aggregation properties of SDS was also found in previous studies with the MARTINI force field. ${ }^{68-69}$

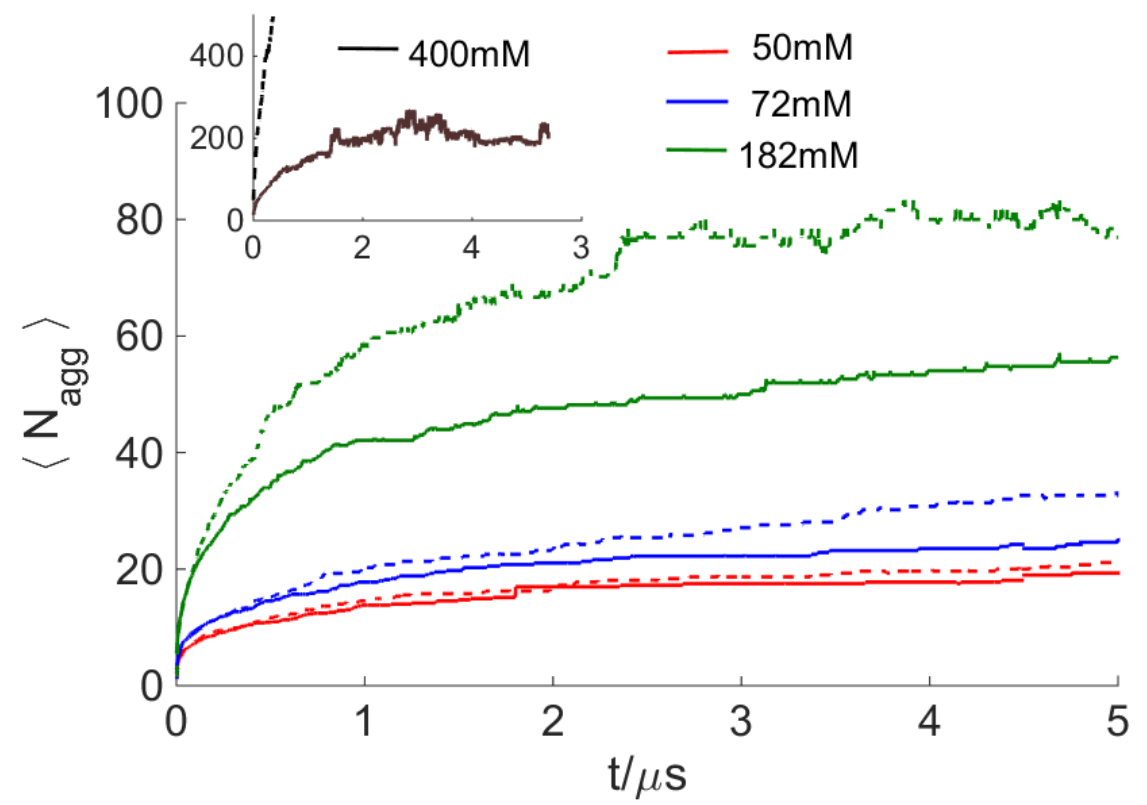

Figure 5. Average aggregation number versus time for $\mathrm{C}_{\mathrm{SDS}}=50 \mathrm{mM}, 72 \mathrm{mM}$, and $182 \mathrm{mM}$. $\left\langle N_{a g g}\right\rangle$ is plotted for each concentration at $\varepsilon_{r}=45$ (dashed lines), and $\varepsilon_{r}=80$ (solid lines). The inset shows the aggregation behaviour at high SDS concentration $\mathrm{C}_{\mathrm{SDS}}=400 \mathrm{mM}$.

The aggregation mechanism of SDS observed in our simulations is in qualitative agreement with the kinetic mechanism proposed by Lund et al. ${ }^{71}$ First individual SDS units rapidly accumulate to form small assemblies. The fast depletion of monomeric SDS from the solution leads to formation of rather regular spherical micelles, of diameter $0.3 \mathrm{~nm}-0.4 \mathrm{~nm}$, corresponding to roughly twice the contour length of a single SDS molecule. At this point, further growth of the micelle occurs by fusion of such aggregates rather than by accumulation of individual SDS units. The growth produces prolate spheroidal micelles that eventually fuse to form nano-tubular structures. This mechanism can be quantified following the evolution of the asphericity parameter $\delta$ of the micelle introduced by Rudnick and Gaspari. ${ }^{72}$ This value is bounded between 0 and 1, with $\delta$ taking the values close to 0 for spherical assemblies, and reaching 1 if all the particles lie on a line (see SI). 


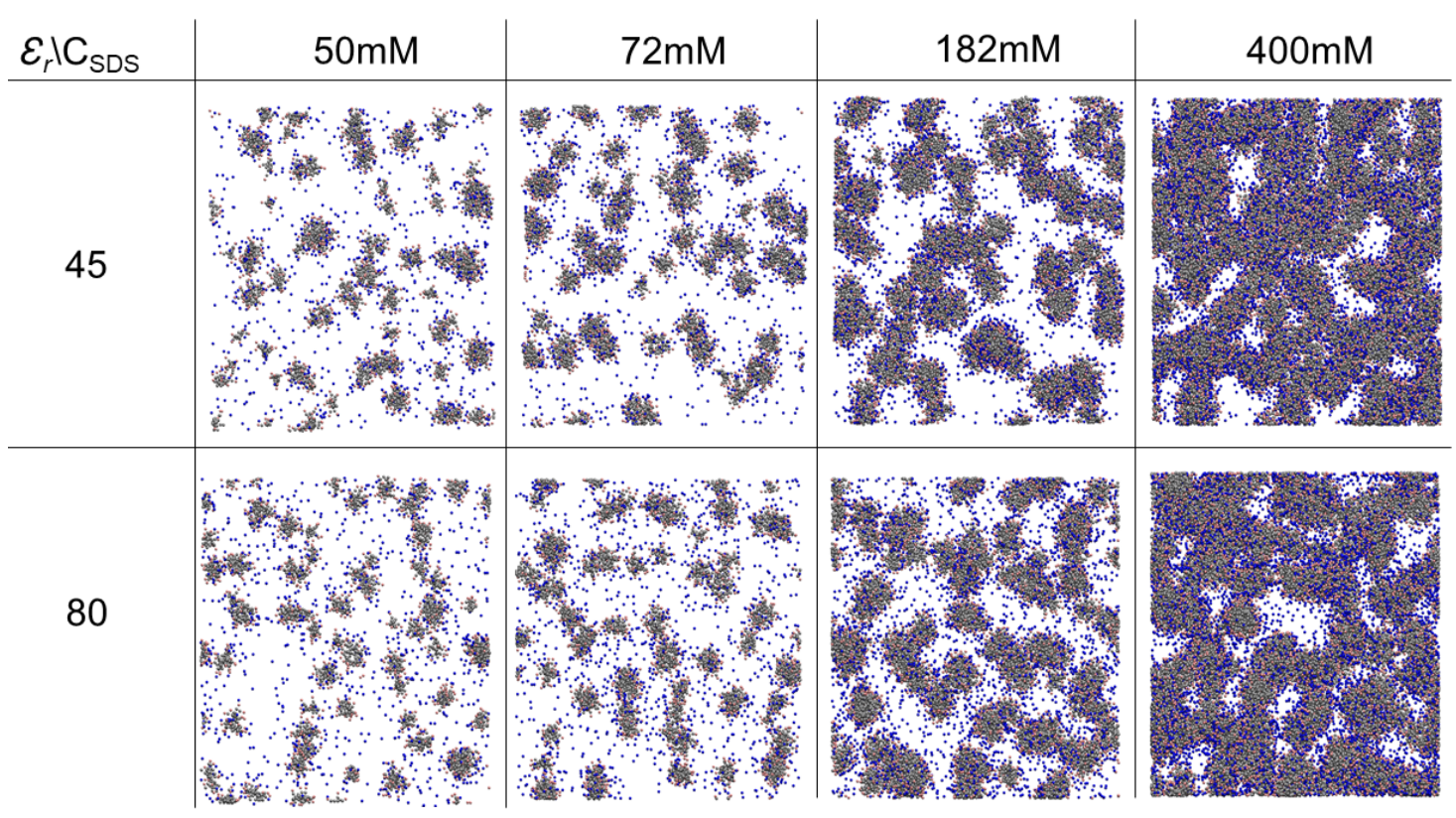

Figure 6. Final snapshots taken after $5 \mu \mathrm{s}$ of the simulations for $\mathrm{C}_{\mathrm{SDS}}=50 \mathrm{mM}, 72 \mathrm{mM}, 182 \mathrm{mM}$, and $400 \mathrm{mM}$ at $\varepsilon_{r}=45$ and 80 . Water is not shown for visual clarity.

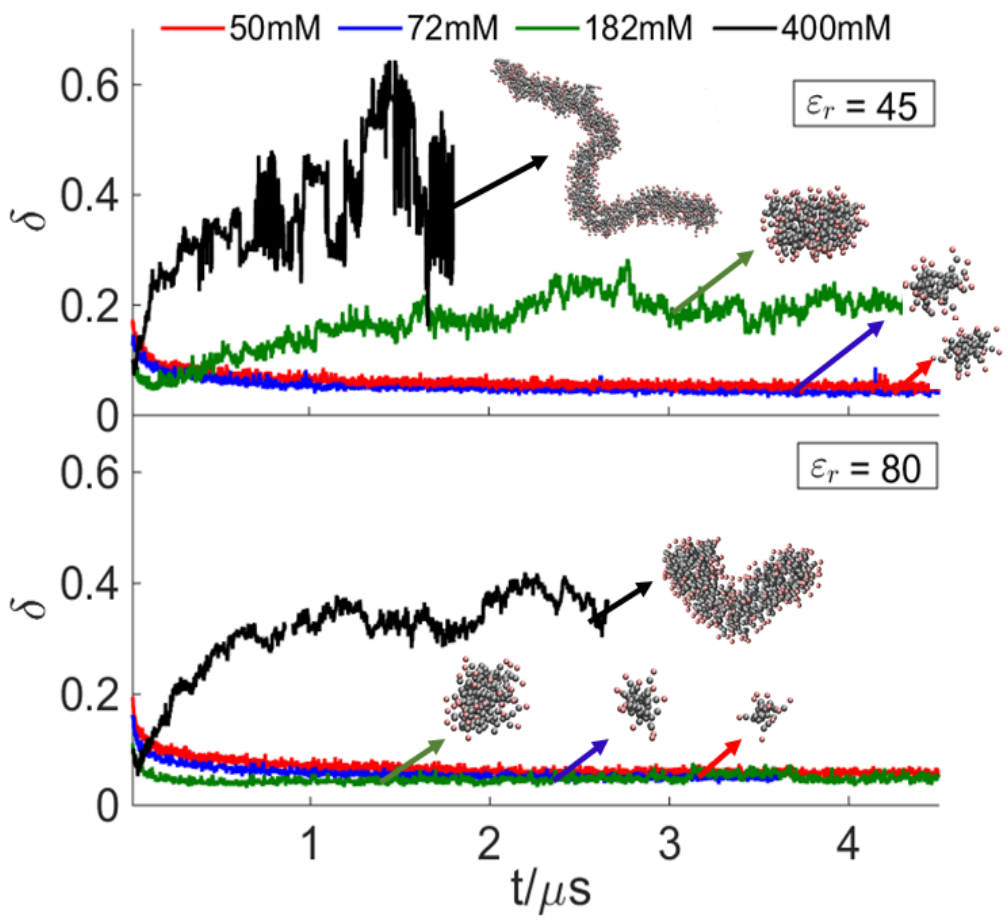

Figure 7. Asphericity parameter $\delta$ with respect to time for $\mathrm{C}_{\mathrm{SDS}}=50 \mathrm{mM}$, $72 \mathrm{mM}, 182 \mathrm{mM}$, and $400 \mathrm{mM}$ at $\varepsilon_{\mathrm{r}}=45$ (top panel) and $\varepsilon_{\mathrm{r}}=80$ (bottom panel). 
Regardless of the dielectric, at all concentrations $\delta$ rapidly develops to values close to 0 , indicating the initial formation of regular spherical structures (Figure 7); for higher concentrations, with $\varepsilon_{r}=45, \delta$ increases over time, signaling the metamorphosis from spherical to spheroidal $\left(\delta \sim 0.2, \mathrm{C}_{\mathrm{SDS}}=182\right.$ $\mathrm{mM})$ or nano-tubular aggregates $\left(\delta \sim 0.4, \mathrm{C}_{\mathrm{SDS}}=400 \mathrm{mM}\right)$. This phenomenon occurs by fusion of smaller spherical units rather than by continuous aggregation of monomeric SDS. The mechanism of fusion into tubular moieties occurs at the poles of the prolate axis of the SDS micelle, as also proposed by Lund et al. ${ }^{71,73}$

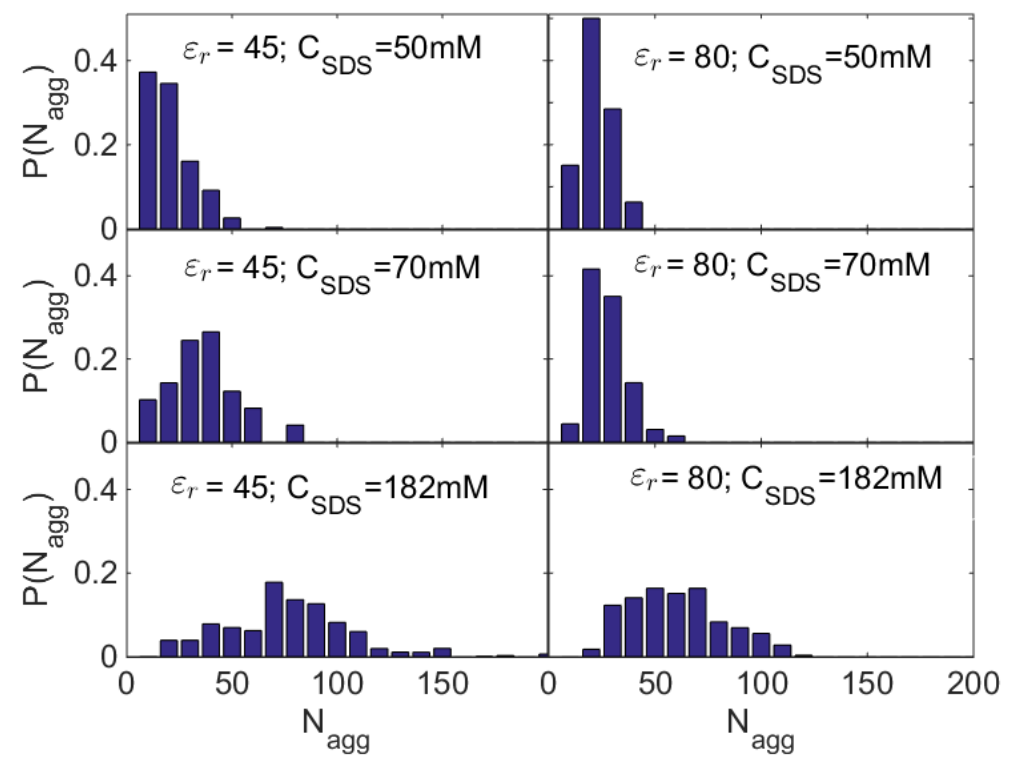

Figure 8. Probability distribution of number of micelles versus micelle size for different concentrations of SDS and value of the relative dielectric constant.

The average aggregation number of the spherical SDS micelles predicted by our model is somewhat smaller than the experimentally measured one $\left(N_{\text {agg }}=54\right.$ for $\mathrm{C}_{\mathrm{SDS}}=50 \mathrm{mM}$, and $N_{\text {agg }}=68$ for $\mathrm{C}_{\mathrm{SDS}}=$ $72 \mathrm{mM}) .{ }^{68}$ In fact, our samples were constituted by a very broad dispersion of micelles of different size, as evident from Figure 8 including micelles as big as those reported by the experiment, which formed in the last microseconds of simulations. It is thus likely that the smaller sizes of the micelles that we observed is a consequence of both the initial conditions (random dispersion of SDS) very far from the thermodynamic equilibrium, and the relatively short sampling time, which promotes the formation of smaller metastable micelles, which may require some activation, in order to fuse into larger aggregates.

\section{Final remarks and conclusion}

This work showed how our new implementation of grid-based electrostatic solvers for particle-field simulations can be used to investigate realistic models of polyelectrolyte soft matter systems. In 
particular, we demonstrated that the hPF-MD approach is capable of treating amphiphilic systems either in extended aggregates, like a lipid bilayer, or in finite micellar forms. In particular, the model predicts in a qualitatively correct manner the effect of charge interactions over the morphology of the aggregates in terms of either area per lipid in bilayers, or shape and size of micelle aggregates. The predicted results are affected by the appropriate choice of the relative dielectric constant, as previously reported in other coarse-grained simulations. In fact, the behavior of these systems is dominated by the formation of ion pairs and their stability, which strongly depends on the relative hydration of the region where they occur.

The behavior of the two POPG and SDS systems is influenced by the choice of the relative dielectric constant in different ways. In particular, the assembly of the POPG bilayer seems to be loosely influenced by the choice of $\varepsilon_{r}$, yielding very good results already at relatively low values of $\varepsilon_{r}$. On the contrary, the assembly dynamics and the stability of different phases of SDS seem to be strongly dependent on the values of $\varepsilon_{r}$, and requiring relatively high $\varepsilon_{r}$ values to avoid the appearance of gelatinous phases that are inconsistent with experiment. Thus, depending on the system and the property of interest, different values of $\varepsilon_{r}$ should be used. This is a common problem encountered in coarse grained molecular dynamics simulations when using a uniform dielectric in systems with multiple phase. Estimates of the effective dielectric corresponding to specific experimental conditions may be either calibrated on specific properties of interest, or derived by particle models as proposed in the literature. ${ }^{74,75}$

The ability of hPF models to treat explicit electrostatics in good agreement with experiment opens up the possibility of simulating efficiently biological moieties where the polyelectrolyte character is dominant; for example, combining it to proposed models for polypeptides with explicit electrostatics. ${ }^{76-}$ 79 Finally, our analysis points to the importance of carefully calibrating $\varepsilon_{r}$ in studies that aim to determine dynamic or structural properties of soft matter assemblies, especially in very specific thermodynamic conditions.

\section{Acknowledgment}

This work received the support of the Norwegian Research Council through the CoE Hylleraas Centre for Quantum Molecular Sciences (Grant No. 262695), and by the Norwegian Supercomputing Program (NOTUR) (Grant No. NN4654K). HBK received funding from the European Union's Horizon 2020 research and innovation programme under the Marie Skłodowska-Curie grant agreement No. 704491. GM and ADN wish to thank the HPC team of Enea (http://www.enea.it) for using the ENEA-GRID and the HPC facilities CRESCO (http://www.cresco.enea.it) in Portici. KS, GD, JG, and MC acknowledge financial support by the Deutsche Forschungsgemeinschaft via the TRR 146 "Multiscale 
Simulation Methods for Soft Matter Systems".

\section{References}

(1) Seidel, C. Physics of amphiphiles: Micelles, vesicles and microemulsions.; North Holland Physics Publishing: Amsterdam/Oxford/New York/Tokyo, 1986; Vol. 37.

(2) Gerhard Gompper, M. S., Cyril Domb, Joel L. Lebowitz In Phase Transitions and Critical Phenomena; Academic Press: 1994; Vol. 16.

(3) Velonia, K.; Cornelissen, J. J. L. M.; Feiters, M. C.; Rowan, A. E.; Nolte, R. J. M. In Nanoscale Assembly: Chemical Techniques; Huck, W. T. S., Ed.; Springer US: Boston, MA, 2005, p 119-185.

(4) Lombardo, D.; Kiselev, M. A.; Magazù, S.; Calandra, P. Amphiphiles Self-Assembly: Basic Concepts and Future Perspectives of Supramolecular Approaches Adv. Cond. Matter Phys. 2015, 2015 , 151683.

(5) Salim, M.; Minamikawa, H.; Sugimura, A.; Hashim, R. Amphiphilic designer nano-carriers for controlled release: from drug delivery to diagnostics MedChemComm 2014, 5, 1602-1618.

(6) Wang, F.; Wen, M.; Feng, K.; Liang, W.-J.; Li, X.-B.; Chen, B.; Tung, C.-H.; Wu, L.-Z. Amphiphilic polymeric micelles as microreactors: improving the photocatalytic hydrogen production of the [FeFe]-hydrogenase mimic in water Chem. Commun. 2016, 52, 457-460.

(7) Chu, B.; Liu, T. Characterization of Nanoparticles by Scattering Techniques J. Nanopart. Res. 2000, 2, 29-41.

(8) Chen, S. H. Small Angle Neutron Scattering Studies of the Structure and Interaction in Micellar and Microemulsion Systems Annu. Rev. Phys. Chem. 1986, 37, 351-399.

(9) Hamley, I. W.; Pedersen, J. S.; Booth, C.; Nace, V. M. A Small-Angle Neutron Scattering Study of Spherical and Wormlike Micelles Formed by Poly(oxyethylene)-Based Diblock Copolymers Langmuir 2001, 17, 6386-6388.

(10) Koch, M. H. J.; Vachette, P.; Svergun, D. I. Small-angle scattering: a view on the properties, structures and structural changes of biological macromolecules in solution $Q$. Rev. Biophys. 2003, 36, 147-227.

(11) Mertens, H. D. T.; Svergun, D. I. Structural characterization of proteins and complexes using small-angle X-ray solution scattering J. Struct. Biol. 2010, 172, 128-141.

(12) Schmölzer, S.; Gräbner, D.; Gradzielski, M.; Narayanan, T. Millisecond-Range Time-Resolved Small-Angle X-Ray Scattering Studies of Micellar Transformations Phys. Rev. Lett. 2002, 88, 258301.

(13) Di Cola, E.; Grillo, I.; Ristori, S. Small Angle X-ray and Neutron Scattering: Powerful Tools for Studying the Structure of Drug-Loaded Liposomes Pharmaceutics 2016, 8, 10.

(14) Seddon, J. M.; Templer, R. H. In Handbook of Biological Physics; Lipowsky, R., Sackmann, E., Eds.; North-Holland: 1995; Vol. 1, p 97-160.

(15) Gutberlet, J. K. T. Lipid Bilayers; Springer-Verlag Berlin Heidelberg, 2001.

(16) Engelman, D. M. Membranes are more mosaic than fluid Nature 2005, 438, 578.

(17) van Meer, G.; Voelker, D. R.; Feigenson, G. W. Membrane lipids: where they are and how they behave Nat. Rev. Mol. Cell Biol. 2008, 9, 112.

(18) Lee, E. H.; Hsin, J.; Sotomayor, M.; Comellas, G.; Schulten, K. Discovery Through the Computational Microscope Structure, 17, 1295-1306.

(19) Lyubartsev, A. P. Multiscale modeling of lipids and lipid bilayers Eur. Biophys. J. 2005, 35, 53. (20) Gunsteren, W. F. v.; Bakowies, D.; Baron, R.; Chandrasekhar, I.; Christen, M.; Daura, X.; Gee, P.; Geerke, D. P.; Glättli, A.; Hünenberger, P. H.; Kastenholz, M. A.; Oostenbrink, C.; Schenk, M.; Trzesniak, D.; Vegt, N. F. A. v. d.; Yu, H. B. Biomolecular Modeling: Goals, Problems, Perspectives Angew. Chem. Int. Edit. 2006, 45, 4064-4092.

(21) Jena, B. P. In Method. Cell Biol.; Academic Press: 2008; Vol. 90, p 19-37. 
(22) Sapay, N.; Tieleman, D. P. In Curr. Top. Membr.; Feller, S. E., Ed.; Academic Press: 2008; Vol. 60, p 111-130.

(23) Lyubartsev, A. P.; Rabinovich, A. L. Recent development in computer simulations of lipid bilayers Soft Matter 2011, 7, 25-39.

(24) Monticelli, L.; Tieleman, D. P. In Biomolecular Simulations: Methods and Protocols; Monticelli, L., Salonen, E., Eds.; Humana Press: Totowa, NJ, 2013, p 197-213.

(25) Marrink, S. J.; de Vries, A. H.; Mark, A. E. Coarse Grained Model for Semiquantitative Lipid Simulations J. Phys. Chem. B 2004, 108, 750-760.

(26) Izvekov, S.; Voth, G. A. A Multiscale Coarse-Graining Method for Biomolecular Systems The Journal of Physical Chemistry B 2005, 109, 2469-2473.

(27) Müller, M.; Katsov, K.; Schick, M. Biological and synthetic membranes: What can be learned from a coarse-grained description? Phys. Rep. 2006, 434, 113-176.

(28) Monticelli, L.; Kandasamy, S. K.; Periole, X.; Larson, R. G.; Tieleman, D. P.; Marrink, S.-J. The MARTINI Coarse-Grained Force Field: Extension to Proteins J. Chem. Theory Comput. 2008, 4, 819-834.

(29) Marrink, S. J.; de Vries, A. H.; Tieleman, D. P. Lipids on the move: Simulations of membrane pores, domains, stalks and curves Biochim. Biophys. Acta 2009, 1788, 149-168.

(30) Murtola, T.; Bunker, A.; Vattulainen, I.; Deserno, M.; Karttunen, M. Multiscale modeling of emergent materials: biological and soft matter Phys. Chem. Chem. Phys. 2009, 11, 1869-1892.

(31) Zun-Jing, W.; Markus, D. Systematic implicit solvent coarse-graining of bilayer membranes: lipid and phase transferability of the force field New J. Phys. 2010, 12, 095004.

(32) Loverde, S. M.; Pantano, D. A.; Christian, D. A.; Mahmud, A.; Klein, M. L.; Discher, D. E. Curvature, rigidity, and pattern formation in functional polymer micelles and vesicles - From dynamic visualization to molecular simulation Curr. Opin. Solid State Mater. Sci. 2011, 15, 277-284.

(33) Piggot, T. J.; Piñeiro, Á.; Khalid, S. Molecular Dynamics Simulations of Phosphatidylcholine Membranes: A Comparative Force Field Study J. Chem. Theory Comput. 2012, 8, 4593-4609.

(34) Srivastava, A.; Voth, G. A. Hybrid Approach for Highly Coarse-Grained Lipid Bilayer Models J. Chem. Theory Comput. 2013, 9, 750-765.

(35) Arnarez, C.; Uusitalo, J. J.; Masman, M. F.; Ingólfsson, H. I.; de Jong, D. H.; Melo, M. N.; Periole, X.; de Vries, A. H.; Marrink, S. J. Dry Martini, a Coarse-Grained Force Field for Lipid Membrane Simulations with Implicit Solvent J. Chem. Theory Comput. 2015, 11, 260-275.

(36) Soares, T. A.; Vanni, S.; Milano, G.; Cascella, M. Toward Chemically Resolved Computer Simulations of Dynamics and Remodeling of Biological Membranes J. Phys. Chem. Lett. 2017, 8, 3586-3594.

(37) Ingólfsson, H. I.; Lopez, C. A.; Uusitalo, J. J.; Jong, D. H. d.; Gopal, S. M.; Periole, X.; Marrink, S. J. The power of coarse graining in biomolecular simulations WIRES Comput. Mol. Sci. 2014, 4, 225-248.

(38) Klein, M. L.; Shinoda, W. Large-Scale Molecular Dynamics Simulations of Self-Assembling Systems Science 2008, 321, 798-800.

(39) Marrink, S. J.; Mark, A. E. Molecular Dynamics Simulation of the Formation, Structure, and Dynamics of Small Phospholipid Vesicles J. Amer. Chem. Soc. 2003, 125, 15233-15242.

(40) Daoulas, K. C.; Muller, M. Single chain in mean field simulations: Quasi-instantaneous field approximation and quantitative comparison with Monte Carlo simulations J. Chem. Phys. 2006, 125, 184904.

(41) Daoulas, K. C.; Muller, M.; de Pablo, J. J.; Nealey, P. F.; Smith, G. D. Morphology of multicomponent polymer systems: single chain in mean field simulation studies Soft Matter 2006, 2, 573583.

(42) Daoulas, K. C.; Cavallo, A.; Shenhar, R.; Muller, M. Phase behaviour of quasi-block copolymers: A DFT-based Monte-Carlo study Soft Matter 2009, 5, 4499-4509. 
(43) Milano, G.; Kawakatsu, T. Hybrid particle-field molecular dynamics simulations for dense polymer systems J. Chem. Phys. 2009, 130, 214106.

(44) Milano, G.; Kawakatsu, T. Pressure calculation in hybrid particle-field simulations J. Chem. Phys. 2010, 133, 214102.

(45) De Nicola, A.; Zhao, Y.; Kawakatsu, T.; Roccatano, D.; Milano, G. Hybrid Particle-Field Coarse-Grained Models for Biological Phospholipids J. Chem. Theory Comput. 2011, 7, 2947-2962.

(46) De Nicola, A.; Zhao, Y.; Kawakatsu, T.; Roccatano, D.; Milano, G. Validation of a hybrid MDSCF coarse-grained model for DPPC in non-lamellar phases Theor. Chem. Acc. 2012, 131, 1167.

(47) Zhao, Y.; Nicola, A. D.; Kawakatsu, T.; Milano, G. Hybrid particle-field molecular dynamics simulations: Parallelization and benchmarks J. Comput. Chem. 2012, 33, 868-880.

(48) De Nicola, A.; Hezaveh, S.; Zhao, Y.; Kawakatsu, T.; Roccatano, D.; Milano, G. Micellar drug nanocarriers and biomembranes: how do they interact? Phys. Chem. Chem. Phys. 2014, 16, 5093-5105. (49) De Nicola, A.; Kawakatsu, T.; Milano, G. Generation of Well-Relaxed All-Atom Models of Large Molecular Weight Polymer Melts: A Hybrid Particle-Continuum Approach Based on ParticleField Molecular Dynamics Simulations J. Chem. Theory Comput. 2014, 10, 5651-5667.

(50) De Nicola, A.; Kawakatsu, T.; Rosano, C.; Celino, M.; Rocco, M.; Milano, G. Self-Assembly of Triton X-100 in Water Solutions: A Multiscale Simulation Study Linking Mesoscale to Atomistic Models J. Chem. Theory Comput. 2015, 11, 4959-4971.

(51) Sevink, G. J. A.; Schmid, F.; Kawakatsu, T.; Milano, G. Combining cell-based hydrodynamics with hybrid particle-field simulations: efficient and realistic simulation of structuring dynamics Soft Matter 2017, 13, 1594-1623.

(52) Zhu, Y.-L.; Lu, Z.-Y.; Milano, G.; Shi, A.-C.; Sun, Z.-Y. Hybrid particle-field molecular dynamics simulation for polyelectrolyte systems Phys. Chem. Chem. Phys. 2016, 18, 9799-9808.

(53) Marrink, S. J.; Risselada, H. J.; Yefimov, S.; Tieleman, D. P.; de Vries, A. H. The MARTINI force field: coarse grained model for biomolecular simulations J. Phys. Chem. B 2007, 111, 7812-7824. (54) Pronk, S.; Páll, S.; Schulz, R.; Larsson, P.; Bjelkmar, P.; Apostolov, R.; Shirts, M. R.; Smith, J. C.; Kasson, P. M.; van der Spoel, D.; Hess, B.; Lindahl, E. GROMACS 4.5: a high-throughput and highly parallel open source molecular simulation toolkit Bioinformatics 2013, 29, 845-854.

(55) Andersen, H. C. Molecular dynamics simulations at constant pressure and/or temperature $J$. Chem. Phys. 1980, 72, 2384-2393.

(56) Jalili, S.; Akhavan, M. A coarse-grained molecular dynamics simulation of a sodium dodecyl sulfate micelle in aqueous solution Colloid. Surface. A 2009, 352, 99-102.

(57) Elmore, D. E. Molecular dynamics simulation of a phosphatidylglycerol membrane FEBS Lett. 2006, 580, 144-148.

(58) Zhao, W.; Róg, T.; Gurtovenko, A. A.; Vattulainen, I.; Karttunen, M. Atomic-Scale Structure and Electrostatics of Anionic Palmitoyloleoylphosphatidylglycerol Lipid Bilayers with $\mathrm{Na}+$ Counterions Biophys. J. 2007, 92, 1114-1124.

(59) Dickey, A.; Faller, R. Examining the Contributions of Lipid Shape and Headgroup Charge on Bilayer Behavior Biophys. J. 2008, 95, 2636-2646.

(60) Kučerka, N.; Holland, B. W.; Gray, C. G.; Tomberli, B.; Katsaras, J. Scattering Density Profile Model of POPG Bilayers As Determined by Molecular Dynamics Simulations and Small-Angle Neutron and X-ray Scattering Experiments J. Phys. Chem. B 2012, 116, 232-239.

(61) Helfrich, W. Elastic Properties of Lipid Bilayers - Theory and Possible Experiments $Z$. Naturforsch. C 1973, 28, 693-703.

(62) Goetz, R.; Gompper, G.; Lipowsky, R. Mobility and elasticity of self-assembled membranes Phys. Rev. Lett. 1999, 82, 221-224.

(63) Lindahl, E.; Edholm, O. Mesoscopic undulations and thickness fluctuations in lipid bilayers from molecular dynamics simulations Biophys. J. 2000, 79, 426-433.

(64) Harmandaris, V. A.; Deserno, M. A novel method for measuring the bending rigidity of model lipid membranes by simulating tethers J. Chem. Phys. 2006, 125, 204905. 
(65) Brandt, E. G.; Braun, A. R.; Sachs, J. N.; Nagle, J. F.; Edholm, O. Interpretation of Fluctuation Spectra in Lipid Bilayer Simulations Biophys. J. 2011, 100, 2104-2111.

(66) Reiss-Husson, F.; Luzzati, V. The Structure of the Micellar Solutions of Some Amphiphilic Compounds in Pure Water as Determined by Absolute Small-Angle X-Ray Scattering Techniques $J$. Phys. Chem. 1964, 68, 3504-3511.

(67) Christov, N. C.; Denkov, N. D.; Kralchevsky, P. A.; Ananthapadmanabhan, K. P.; Lips, A. Synergistic Sphere-to-Rod Micelle Transition in Mixed Solutions of Sodium Dodecyl Sulfate and Cocoamidopropyl Betaine Langmuir 2004, 20, 565-571.

(68) Wang, S.; Larson, R. G. Coarse-Grained Molecular Dynamics Simulation of Self-Assembly and Surface Adsorption of Ionic Surfactants Using an Implicit Water Model Langmuir 2015, 31, 1262 1271.

(69) Velinova, M,; Sengupta, D.; Tadjer, A. V.; Marrink, S.-J. Sphere-to-Rod Transitions of Nonionic Surfactant Micelles in Aqueous Solution Modeled by Molecular Dynamics Simulations, Langmuir, 2011, 27, 14071-14077.

(70) Anderson, R. L.; Bray, D. J.; Del Regno, A.; Seaton, M. A.; Ferrante, A. S.; Warren, P. B. Micelle formation in alkyl sulfate surfactants using dissipative particle dynamics J. Chem. Theory Comput. 2018, 14, 2633-2643.

(71) Jensen, G. V.; Lund, R.; Gummel, J.; Narayanan, T.; Pedersen, J. S. Monitoring the Transition from Spherical to Polymer-like Surfactant Micelles Using Small-Angle X-Ray Scattering Angew. Chem. Int. Edit. 2014, 53, 11524-11528

(72) Rudnick, J.; Gaspari, G. The Shapes of Random Walks Science 1987, 237, 384-389.

(73) Lund, R.; Willner, L.; Monkenbusch, M.; Panine, P.; Narayanan, T.; Colmenero, J.; Richter, D. Structural Observation and Kinetic Pathway in the Formation of Polymeric Micelles Phys. Rev. Lett. 2009, 102, 188301.

(74) Vasilyev, V. Determination of the Effective Dielectric Constant from the Accurate Solution of the Poisson Equation J. Comput. Chem. 2002, 23, 1254-1265.

(75) Xue, Q. Effective dielectric constant of composite with interfacial shells Physica B Condens. Matter 2004, 344, 129-132.

(76) Cascella, M.; Neri, M. A.; Carloni, P.; Dal Peraro, M. Topologically Based Multipolar Reconstruction of Electrostatic Interactions in Multiscale Simulations of Proteins J. Chem. Theory Comput. 2008, 4, 1378-1385.

(77) Alemani, D.; Collu, F.; Cascella, M.; Dal Peraro, M. A Nonradial Coarse-Grained Potential for Proteins Produces Naturally Stable Secondary Structure Elements J. Chem. Theory Comput. 2010, 6, 315-324.

(78) Spiga, E.; Alemani, D.; Degiacomi, M. T.; Cascella, M.; Dal Peraro, M. ElectrostaticConsistent Coarse-Grained Potentials for Molecular Simulations of Proteins J. Chem. Theory Comput. 2013, 9, 3515-3526.

(79) Bore, S. L.; Milano, G.; Cascella, M. Hybrid Particle-Field Model for Conformational Dynamics of Peptide Chains J. Chem. Theory Comput. 2018, 14, 1120-1130. 
Table of Contents

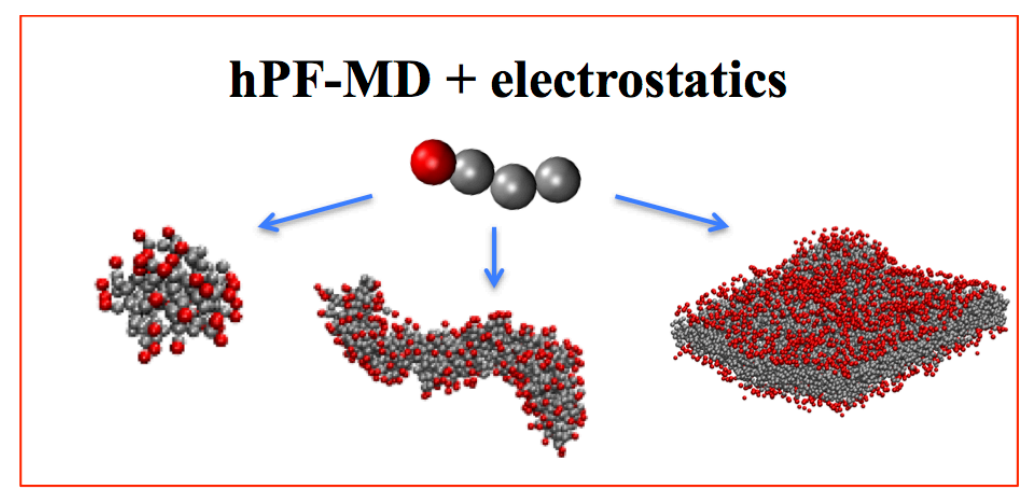

